\title{
Antenna Packaging for In-body Applications
}

\author{
Jordi Romeu, Giselle González-López, Sebastián Blanch, Lluís Jofre, \\ Commsenslab, Dept. Signal Theory and Communications, Universitat Politecnica de Catalunya, UPC, Barcelona, \\ Spain, e-mail romeu@tsc.upc.edu
}

\begin{abstract}
A cylindrical mode expansion of the fields produced by an embedded antenna is used to determine the dimensions of the antenna packaging in order to minimize antenna impedance changes when the antenna is immersed in a varying dielectric medium.
\end{abstract}

Index Terms-RFID, embedded antenna.

\section{INTRODUCTION}

T HE use of embedded antennas for sensing and communication poses the particular problem of designing antennas that can operate efficiently when immersed in a medium. For example, in in-body applications the variations of dielectric constant of different types of tissues imposes antenna designs able to operate when immersed in media with a wide range of values for the dielectric constant, and specifically with high values of dielectric constant. It is well known that an antenna made of perfect conductor immersed in a medium with losses experiments a change in its input impedance [1]. On the other hand, in [2] it is shown that the high reactive fields in the surrounding of the antenna are responsible for high losses when the antenna is immersed in a lossy medium, and the radiated power can be maximized if the antenna is encapsulated in a low-loss dielectric.

In this paper we focus our attention on the packaging requirements, that is the dimensions of the dielectric encapsulation to guarantee a stable antenna impedance when immersed in a dielectric medium. This is of particular importance in RFID based communications. In this case variations of the antenna impedance can produce severe mismatch to the RFID chip and in consequence reducing dramatically the activation range of the RFID chip.

To give general guidelines and have a better understanding of the physical process the field produced by the antenna is expressed in terms of a cylindrical mode expansion. For RFID applications the antennas are small in terms of the wavelength and even the near-fields can be expressed in a reduced number of modes. For each mode the stored energy can be found as function to the distance to the antenna, and the volume of a package that contains most of the reactive near field energy can be determined. In this way antenna impedance will suffer small changes when immersed in a dielectric medium.

The effectiveness of this approach is validated through numerical simulations.

\section{ANTENNA IMPEDANCE MODELLING}

Changes on the antenna impedance produce an antenna impedance mismatch that affects the power transfer to the RFID chip and in consequence reduces its activation range.
RFID antennas have usually highly reactive impedances with relatively low values of the radiation resistance in order to have complex conjugate matching to the RFID chip [3]. It is important to understand how antenna impedance changes when immersed in a medium. Since RFID antennas are highly reactive, being its input reactance of the order of a few hundreds ohms, it is particularly interesting to design the antenna packaging to minimize the changes in the antenna reactance.

Following the definitions of [4], and assuming a time variation of $e^{j \omega t}$ for an antenna immersed in a medium with losses with electric permittivity and magnetic permeability given by

$$
\mu=\mu_{r}-j \mu_{i} ; \epsilon=\epsilon_{r}-j \epsilon_{i}
$$

its input reactance is given by

$$
X_{o}(\omega)=\frac{\omega}{\left|I_{o}(\omega)\right|^{2}} \int_{v_{o}}\left(\mu_{r}|\mathbf{H}|^{2}-\epsilon_{r}|\mathbf{E}|^{2}\right) d v
$$

where $I_{o}$ is the antenna input current, $v_{o}$ is all space excluding the antenna volume and $\mathbf{H}$ and $\mathbf{E}$ are the magnetic and electric fields produced by the antenna. Equation 2 expresses the fact that the antenna reactance depends on the balance between the stored magnetic and electric energy of the fields produced by the antenna. With the usual definitions

$$
W_{m}=\frac{1}{4} \int_{v_{o}} \mu_{r}|\mathbf{H}|^{2} d v ; W_{e}=\frac{1}{4} \int_{v_{o}} \epsilon_{r}|\mathbf{E}|^{2} d v
$$

where $W_{m}$ and $W_{e}$ are the stored magnetic and electric energy, and the electric and magnetic energy densities have the usual form

$$
U_{m}=\frac{1}{4} \mu_{r}|\mathbf{H}|^{2} ; U_{e}=\frac{1}{4} \epsilon_{r}|\mathbf{E}|^{2}
$$

Given that the integration volume extends to the infinity $W_{m}$ and $W_{e}$ are infinity, but their difference has a finite value and

$$
X_{o}(\omega)=\frac{4 \omega}{\left|I_{o}(\omega)\right|^{2}}\left[W_{m}-W_{e}\right]
$$

As matter of fact the electric and magnetic energy associated to the radiation fields is the same, and in consequence only the stored energy associated to the reactive fields needs to be found in order to obtain the antenna reactance. In [5] it is suggested to find the energy density associated to the reactive fields as

$$
U_{e}^{r e a c}=\frac{1}{4} \epsilon_{r}|\mathbf{E}|^{2}-\frac{1}{4 c} R e\left[\mathbf{E} \times \mathbf{H}^{*}\right] \hat{r}=U_{e}-U_{e}^{r a d}
$$

The second term of (6) expresses the fact that electric energy density associated to the radiation fields is one half the power flow given by the Poynting vector $\wp$ divided by the propagation velocity $c$. 
With this general framework let us consider now an antenna whose fields are expressed in terms of cylindrical harmonics. Most antennas used in RFID applications are dipole-like antennas with some type of meandering for size reduction and matching at the feed point to obtain the desired antenna impedance. We will consider the $T M_{z}$ case as it is most representative of usual antennas employed in RFID embedded applications, although the formulation can be easily extended to the $T E_{z}$ case. For a particular mode with indices $n$ and $k_{z}$ the electric and magnetic fields have the following expressions, where the dependence $e^{j n \phi}$ and $e^{-j k_{z}}$ has been omitted.

$$
\begin{aligned}
E_{\rho} & =\frac{j k_{z}}{k} \frac{\partial}{\partial \rho} H_{n}^{(2)}\left(k_{\rho} \rho\right) \\
E_{\phi} & =-\frac{n k_{z}}{k \rho} H_{n}^{(2)}\left(k_{\rho} \rho\right) \\
E_{z} & =-\frac{k_{\rho}^{2}}{k} H_{n}^{(2)}\left(k_{\rho} \rho\right) \\
H_{\rho} & =\frac{n}{\eta \rho} H_{n}^{(2)}\left(k_{\rho} \rho\right) \\
H_{\phi} & =\frac{j}{\eta} \frac{\partial}{\partial \rho} H_{n}^{(2)}\left(k_{\rho} \rho\right) \\
H_{z} & =0
\end{aligned}
$$

where $k_{\rho}^{2}+k_{z}^{2}=k^{2}, \eta=\sqrt{\frac{\mu_{r}}{\epsilon_{r}}}$ and $k=\omega \sqrt{\mu_{r} \epsilon_{r}}$. We assume that the medium is lossless. We can define the wave impedance $Z_{T M}^{+}$for an outwardly propagating wave as

$$
Z_{T M}^{+}=-\frac{E_{z}}{H_{\phi}}=-j \eta \frac{k_{\rho}^{2}}{k} \frac{H_{n}^{(2)}\left(k_{\rho} \rho\right)}{\frac{\partial}{\partial \rho} H_{n}^{(2)}\left(k_{\rho} \rho\right)}
$$

and the electric energy density due to the radiated field will be given by

$$
U_{e}^{r a d}=\frac{1}{4 c} \operatorname{Re}\left[\mathbf{E} \times \mathbf{H}^{*}\right] \hat{\rho}=\frac{1}{4 c}\left|E_{z}\right|^{2} \frac{1}{\operatorname{Re}\left(Z_{T M}^{+}\right)}
$$

which leads to the following expression for the reactive electric energy density

$$
U_{e}^{\text {reac }}=\frac{1}{4} \epsilon_{r}|\mathbf{E}|^{2}-\frac{1}{4 c}\left|E_{z}\right|^{2} \frac{1}{\operatorname{Re}\left(Z_{T M}^{+}\right)}
$$

With the field expressions of (7)

$$
\begin{array}{r}
U_{e}^{\text {reac }}=\frac{1}{4} \epsilon_{r}\left[\left(\frac{k_{z}}{k}\right)^{2}\left|\frac{\partial}{\partial \rho} H_{n}^{(2)}\left(k_{\rho} \rho\right)\right|^{2}+\right. \\
\left.\left.+\left[\left(\frac{n k_{z}}{k \rho}\right)^{2}+\left(\frac{k_{\rho}}{k}\right)^{2}\left(1-\frac{\eta}{\operatorname{Re}\left(Z_{T M}^{+}\right)}\right)\right]\left|H_{n}^{(2)}\left(k_{\rho} \rho\right)\right|^{2}\right]\right]
\end{array}
$$

In fig. 1 the reactive electric energy density given by (11) is plotted for the first modes $n=0,1,2$ and for $k_{z}=0$ for an antenna that fits in cylinder of radius $0.01 \lambda$. The energy density is plotted against the distance expressed in terms of the free space wavelength on the medium. Notice that in this case the plot of fig. 1 is independent of the value of the dielectric constant of the medium. To this end, also the energy density has been arbitrarily normalized to $0 \mathrm{~dB}$ at a distance of $1 \lambda$ for the mode $n=0$. The plot clearly shows that at a distance of $0.1 \lambda$ the reactive energy associated to the modes has strongly decayed. Notice that due to the orthogonality of the modes the stored electric energy is the summation of the energy of each of the modes. The specific magnitude by which each one of the modes will be excited will depend on the particular geometry of the antenna. We can conclude that if an antenna with an electric dipolar behavior is packaged inside a dielectric cylinder of radius of the other of $0.1 \lambda$ the major fraction of the reactive electric fields will be contained inside this cylinder. In consequence it is expected that when the packaged antenna is embedded in media with different dielectric constants, although the change in boundary conditions will modify the field distribution the stored electric energy will not change substantially and according to (5) the antenna reactance will be modified only to a small extent

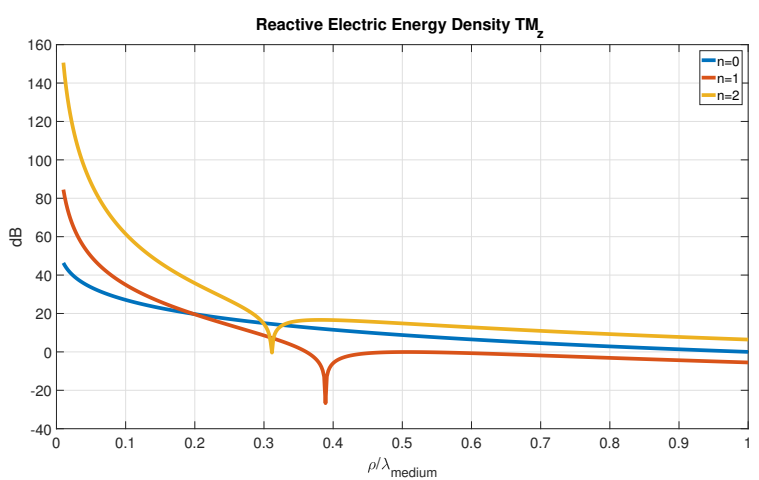

Figure 1: Reactive electric energy density.

The antenna resistance is related with the antenna power dissipation. For a lossless antenna in a lossless medium all power dissipation occurs in the form of radiation. In this case

$$
P_{r}=\frac{1}{2}\left|I_{0}\right|^{2} R_{r}
$$

where $R_{r}$ is the radiation resistance. For an antenna packaged in a dielectric cylinder (medium 1 ) of radius $\rho_{0}$ immersed in medium (medium 2) of changing dielectric constant different from the dielectric constant of the packaging there will be a mismatch between the waves propagating in each one of the media. The reflection coefficient for a $T M_{z}$ mode is

$$
\Gamma_{n}^{T M}\left(\rho=\rho_{0}\right)=\frac{H_{n}^{(2)}\left(k_{\rho 1} \rho_{0}\right)}{H_{n}^{(1)}\left(k_{\rho 1} \rho_{0}\right)} \frac{\eta_{2}-\eta_{1}}{\eta_{2} \frac{Z_{T M}^{+1}}{Z_{T M}^{-1}}+\eta_{1}}
$$

where $Z_{T M}^{+1}$ is the impedance given by eq. 8 and $Z_{T M}^{-1}$ is the wave impedance for an inwardly propagating wave, and

$$
Z_{T M}^{-1}=\left(Z_{T M}^{+1}\right)^{*}
$$

Cylindrical modes of order $n>k a$, where $a$ is the radius of the minimum cylinder that encloses the antenna, are strongly attenuated and do not contribute to the radiation fields. As long as $a<0.16 \lambda$ in the packaging medium only mode with index $n=0$ is involved in radiation. Fig. 2 shows the reflection coefficient for the mode $n=0$ and $k_{z}=0$ from medium with refraction index $n_{1}$ to $n_{2}$ as a function of the radius of the inner shell. For a radius larger that $0.2 \lambda_{1}$ the reflection coefficient can be well approximated by

$$
\Gamma^{T M}=\frac{\eta_{2}-\eta_{1}}{\eta_{2}+\eta_{1}}
$$


The impedance mismatch in changing from medium 1 to

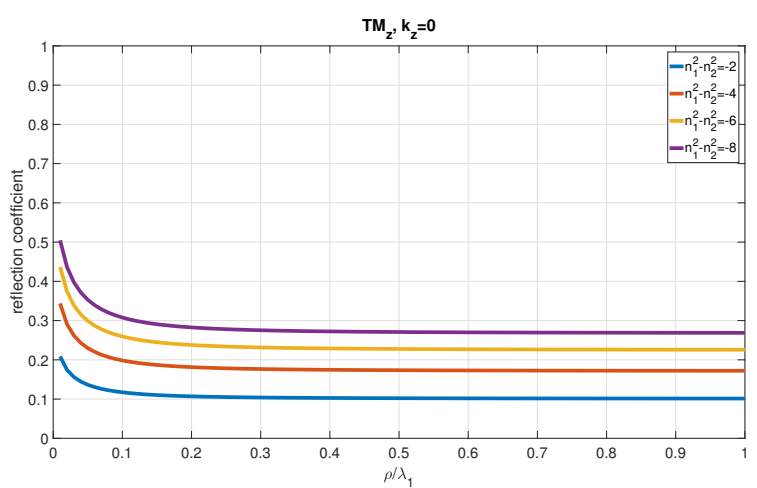

Figure 2: Reflection coefficient.

medium 2 will cause a reflection and a changes in the radiated power. Assuming a constant current on the antenna we can write

$$
P_{r}=\frac{1}{2}\left|I_{0}\right|^{2} R_{r}\left(1-\left|\Gamma_{0}^{T M}\left(\rho=\rho_{0}\right)\right|^{2}\right)=\frac{1}{2}\left|I_{0}\right|^{2} R_{r}^{e}
$$

where $R_{r}^{e}$ is the radiation resistance of the antenna embedded in medium 2 and

$$
\frac{R_{r}^{e}}{R_{r}}=1-\left|\Gamma_{0}^{T M}\left(\rho=\rho_{0}\right)\right|^{2}
$$

\section{NUMERICAL VERIFICATION}

In order to verify the results of the previous section a set of numerical simulations have been conducted with the use of CST Studio design. As a test antenna a dipole of $90 \mathrm{~mm}$ length and a radius of $1 \mathrm{~mm}$ has been simulated at a frequency of $868 \mathrm{MHz}$. The first simulation has been conducted to verify that the electric and magnetic energy densities are confined at very close distance of the antenna. In fig. 1 the electric and magnetic energy of the dipole immersed in a lossless medium with $\epsilon_{r}=4$ are shown. A cylinder of radius $0.1 \lambda_{1}$ enclosing the antenna is also shown. Notice that in this case the total energy, not only the reactive energy is shown. It can be appreciated that actually most of the energy is confined inside the cylinder. To validated the stability of the antenna impedance when it is embedded in a medium of changing dielectric constant the impedance of the dipole at $868 \mathrm{MHz}$ packaged inside a cylinder of $17.28 \mathrm{~mm}$ of radius and length of $70 \mathrm{~mm}$ with dielectric constant $\epsilon_{r}=4$ is shown in fig. 5 . The results clearly show that for medium with a dielectric constant higher than the packaging medium the reactance remains practically constant. On the other hand, as expected the antenna resistance changes due to the mismatch. In fig.4 it is shown the expected variation of the input resistance of an antenna packaged inside a cylinder of radius $0.1 \lambda$ as a function of the contrast with the surrounding medium. The curves are the result of finding the reflection coefficient for the mode as in (13) or with the approximate expression (15) and the are compared with the resistance simulated results by CST. The resistance change in simulations is larger that the one predicted from the modal approach. The modal approach provides a better estimate than the far-field approximation. It

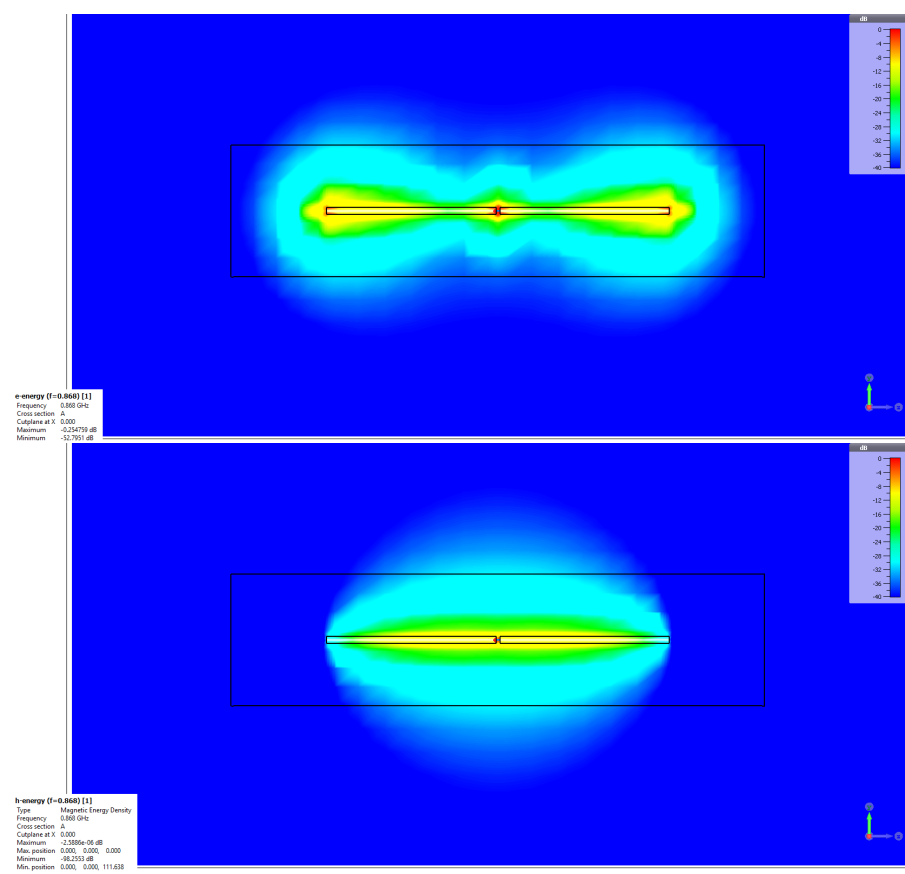

Figure 3: Energy densities electric (top), magnetic (bottom) for a dipole immersed in a medium of $\epsilon_{r}=4$

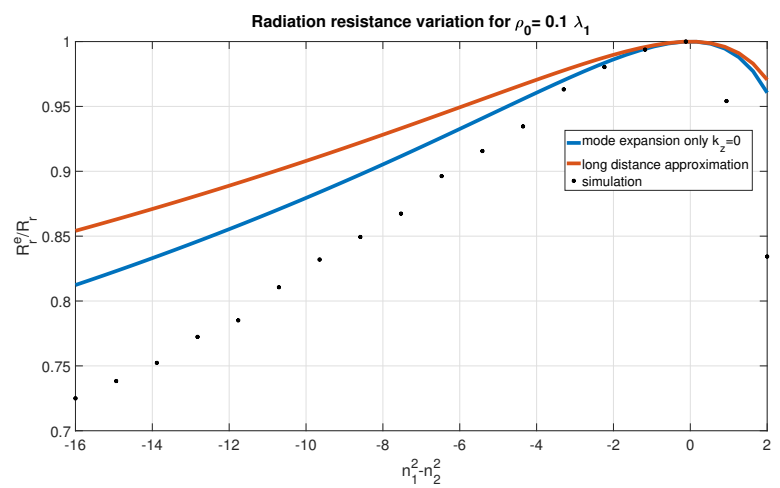

Figure 4: Resistance variation.

must be noted that in the modal approach only the reflection coefficient for the mode $k_{z}=0$ has been taken into account, while it is clear than in the case of a short antenna there will be a wide spectral content along $k_{z}$. It can be concluded that using a cylindrical packaging of $0.1 \lambda$ radius in the packaging medium provides a good isolation from the impedance point of view with respect a changing embedding medium. It can be observed that when the dielectric constant of the packaging is smaller that the embedding medium the effect of packaging actually stabilizes the value of the antenna reactance, while inevitably the mismatch produced by the changing medium produces some changes in the resistance.

\section{ApPlication to RFID ANTENNA DESIGN}

To illustrate the effectiveness of the presented approach, the effect of the packaging of an RFID antenna designed to operate in a changing biological medium is presented. The antenna in fig. 6 , with resonance frequency at $868 \mathrm{MHz}$, has 


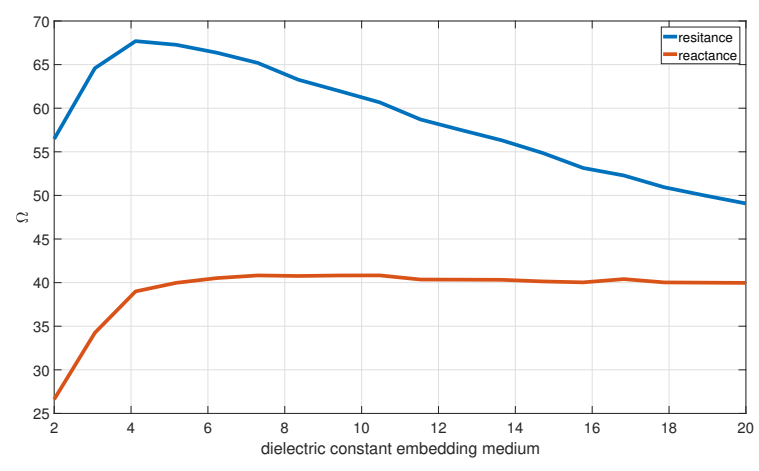

Figure 5: Variation of the antenna impedance when embedded in a medium of varying dielectric constant.

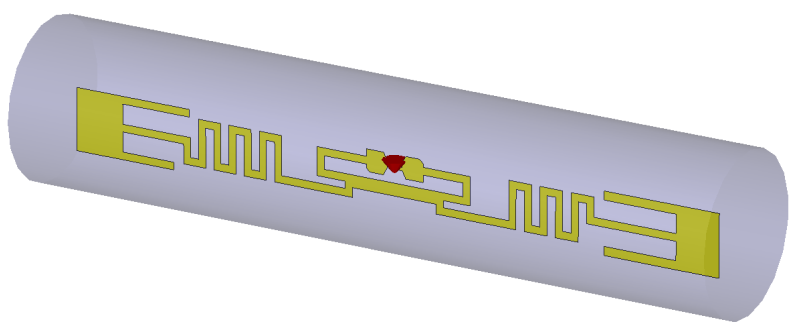

Figure 6: Modeled RFID Antenna.

been modeled using CST [6], [7]. The behaviour of its input impedance has been analysed for frequencies between 0.8 and $0.9 \mathrm{GHz}$, for three scenarios: (a) no Packaging, (b) $r_{p}=0.1 \lambda_{p}$ $=10.9 \mathrm{~mm}$ radius Packaging and (c) $r_{p}=0.5 \lambda_{p}=54.6 \mathrm{~mm}$ radius Packaging. These results are presented in Figures 7 to 9. We have considered as packaging the ABS1000 filament from PREPERM $\left(\varepsilon_{r}=10, \tan \delta=0.004\right)$ [8].

For every case, it has been considered that the antenna is embedded in a medium with different relative permittivity $\left(\varepsilon_{r}\right)$ representative of different biological medium. Three different tissues have been considered: brain, bone and fat [9].

From fig. 7, it is possible to notice that the input impedance of the simulated antenna highly varies as the dielectric properties of the embedding medium change. While for high permittivity values the input impedance presents a very high real and imaginary part, for smaller relative permittivities they both reduce considerably and exhibit impedance values closer (in the Smith Chart) to those typical of RFID antennas (see Table I).

By placing a small insulation in the shape of a cylindrical packaging of radius $r_{p}$ (dependent on $\lambda_{p}$, which is the effective wavelength of the packaging material), the effect of the change in the relative permittivity of the surrounding medium is mitigated (Fig. 8 and 9). In Figures 8 and 9, a cylindrical packaging of $r_{p}=0.1 \lambda_{p}$ and $0.5 \lambda_{p}$ respectively, is considered. It can be noticed that by further increasing the radius of the insulation, it is possible to reduce the effect of the change

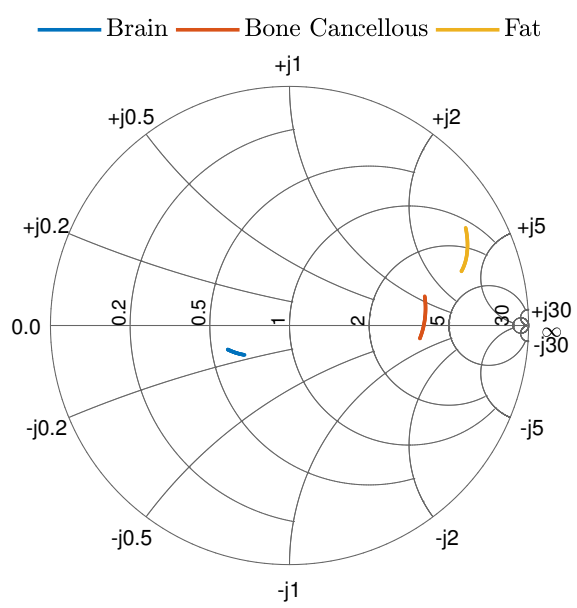

Figure 7: Antenna Impedance from $0.8 \mathrm{GHz}$ to $0.9 \mathrm{GHz}$. No Packaging. Dielectric properties: Brain $\left(\varepsilon_{r}=57, \tan \delta=0.6\right)$, Bone Cancellous $\left(\varepsilon_{r}=20.9, \tan \delta=0.32\right)$, Fat $\left(\varepsilon_{r}=11.3\right.$, $\tan \delta=0.2$ )

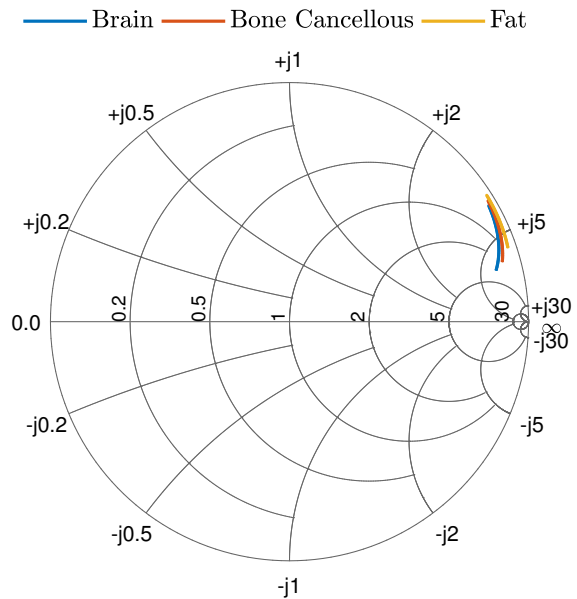

Figure 8: Antenna Impedance from $0.8 \mathrm{GHz}$ to $0.9 \mathrm{GHz}$. Packaging radius $r_{p}=0.1 \lambda_{p}=10.9 \mathrm{~mm}$. Dielectric properties: Brain $\left(\varepsilon_{r}=57, \tan \delta=0.6\right)$, Bone Cancellous $\left(\varepsilon_{r}=20.9\right.$, $\tan \delta=0.32)$, Fat $\left(\varepsilon_{r}=11.3, \tan \delta=0.2\right)$.

in the relative permittivity of the medium until it is almost neglectable.

\section{CONCLUSiOnS}

By considering the associated energy of cylindrical radiation modes in the vicinity of an antenna a proper packaging can be designed to minimize the antenna reactance changes due to changes in the dielectric medium. For dipole-like antennas small in terms of the wavelength the $T M_{z}$ mode expansion is enough to model the antenna behavior and the first few modes sufficient to show that a packaging of a radius of $0.1 \lambda_{p}$ contains most of the stored reactive energy. Through simulations it is shown that for a realistic RFID antenna embedded 


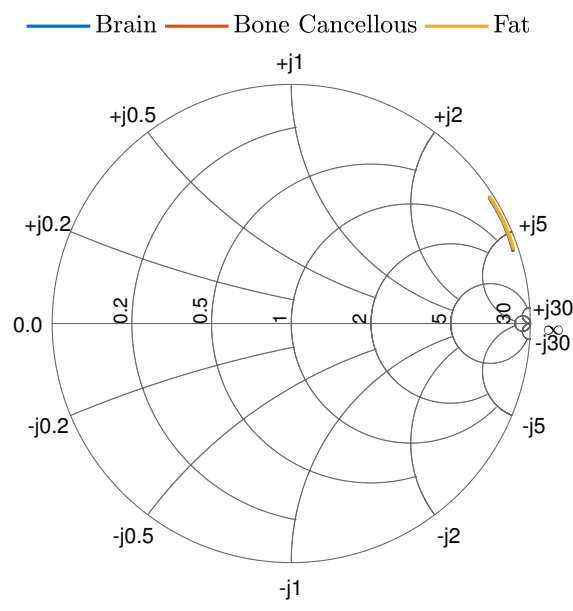

Figure 9: Antenna Impedance from $0.8 \mathrm{GHz}$ to $0.9 \mathrm{GHz}$. Packaging radius $r_{p}=0.5 \lambda_{p}=54.6 \mathrm{~mm}$. Dielectric properties: Brain $\left(\varepsilon_{r}=57, \tan \delta=0.6\right)$, Bone Cancellous $\left(\varepsilon_{r}=20.9\right.$, $\tan \delta=0.32)$, Fat $\left(\varepsilon_{r}=11.3, \tan \delta=0.2\right)$.

Table I: Input impedance at $0.868 \mathrm{GHz}$ of the simulated RFID Antenna.

\begin{tabular}{|l|c|c|c|}
\hline & $\begin{array}{c}\text { Brain } \\
\varepsilon_{r}=57 \\
\tan \delta=0.6\end{array}$ & $\begin{array}{c}\text { Bone } \\
\text { Cancellous } \\
\varepsilon_{r}=20.9 \\
\tan \delta=0.32\end{array}$ & $\begin{array}{c}\text { Fat } \\
\varepsilon_{r}=11.3 \\
\tan \delta=0.2\end{array}$ \\
\hline $\begin{array}{c}\text { No } \\
\text { Packaging }\end{array}$ & $30.1-j 7$ & $178.5+j 2.2$ & $128.3+j 191$ \\
\hline $\begin{array}{l}\text { Packaging } \\
r_{p}=0.1 \lambda_{p}\end{array}$ & $56.6+j 272.3$ & $35+j 258.7$ & $16.2+j 236.9$ \\
\hline $\begin{array}{l}\text { Packaging } \\
r_{p}=0.5 \lambda_{p}\end{array}$ & $11.7+j 242.6$ & $11.5+j 239.3$ & $9.6+j 239.2$ \\
\hline
\end{tabular}

in different biological media the antenna reactance can be stabilized and as the diameter of the packaging increases the value tends to be more stable. On the other hand, the antenna resistance inevitably changes when the medium changes due to the reflection that occurs at the interface between the packaging and the medium.

Further experimental verification, as well as system assessment performance of the proposed packaging is still needed, but the proposed design approach is general enough to approach antenna packaging design for embedded antennas that need to operate in different or changing media.

\section{ACKNOWLEDGMENT}

This work was supported by the Spanish "Comision Interministerial de Ciencia y Tecnologia" (CICYT) under projects TEC2016-78028-C3-1P, MDM2016-O6OO, and Catalan Research Group 2017 SGR 219.

\section{REFERENCES}

[1] G. Deschamps, "Impedance of an antenna in a conducting medium," IRE Transactions on Antennas and Propagation, vol. 10, no. 5, pp. 648-650, Sep. 1962.
[2] A. K. Skrivervik, M. Bosiljevac, and Z. Sipus, "Fundamental limits for implanted antennas: Maximum power density reaching free space," IEEE Transactions on Antennas and Propagation, vol. 67, no. 8, pp. 4978 4988, Aug 2019.

[3] K. V. S. Rao, P. V. Nikitin, and S. F. Lam, "Antenna design for uhf rfid tags: a review and a practical application," IEEE Transactions on Antennas and Propagation, vol. 53, no. 12, pp. 3870-3876, Dec 2005.

[4] A. D. Yaghjian and S. R. Best, "Impedance, bandwidth, and q of antennas," IEEE Transactions on Antennas and Propagation, vol. 53, no. 4, pp. 1298-1324, April 2005.

[5] R. Collin and S. Rothschild, "Evaluation of antenna q," IEEE Transaction on Antennas and Propagation, vol. 12, no. 1, pp. 23-27, January 1964.

[6] [Online]. Available: https://www.alientechnology.com/products/tags/ squiggle/

[7] S. Shao, R. J. Burkholder, and J. L. Volakis, "Design approach for robust uhf rfid tag antennas mounted on a plurality of dielectric surfaces [antenna designer's notebook]," IEEE Antennas and Propagation Magazine, vol. 56, no. 5, pp. 158-166, 2014.

[8] [Online]. Available: https://www.preperm.com/webshop/productcategory/3d-filaments/

[9] [Online]. Available: https://itis.swiss/virtual-population/tissueproperties/database/dielectric-properties/ 